\title{
Pre-implant magnetic resonance and transrectal ultrasound imaging in high-dose-rate prostate brachytherapy: comparison of prostate volumes, craniocaudal extents, and contours
}

\author{
Simone Grisotto, PhD', Annamaria Cerrotta, MD², Brigida Pappalardi, MD², Mauro Carrara, PhD', Antonella Messina, MD³. \\ Chiara Tenconi, PhD', Prof. Riccardo Valdagni, MD ${ }^{4,5,6}$, Carlo Fallai, MD² \\ 'Medical Physics Unit, Fondazione IRCCS Istituto Nazionale dei Tumori, Milan, ${ }^{2}$ Radiotherapy 2 Unit, Fondazione IRCCS Istituto Nazionale \\ dei Tumori, Milan, ${ }^{3}$ Diagnostic Imaging Unit, Fondazione IRCCS Istituto Nazionale dei Tumori, Milan, ${ }^{4}$ Radiotherapy I Unit, Fondazione \\ IRCCS Istituto Nazionale dei Tumori, Milan, ${ }^{5}$ Prostate Program, Scientific Directorate, Fondazione IRCCS Istituto Nazionale dei Tumori, Milan \\ ${ }^{6}$ Department of Medicine and Surgery, Universita' degli Studi, Milan, Italy
}

\begin{abstract}
Purpose: The purpose of this study was to compare the prostate contours drawn by two radiation oncologists and one radiologist on magnetic resonance (MR) and transrectal ultrasound (TRUS) images. TRUS intra- and inter-fraction variability as well as TRUS vs. MR inter-modality and inter-operator variability were studied.

Material and methods: Thirty patients affected by localized prostate cancer and treated with interstitial high-doserate (HDR) prostate brachytherapy at the National Cancer Institute in Milan were included in this study. Twenty-five patients received an exclusive two-fraction $(14 \mathrm{~Gy} /$ fraction) treatment, while the other 5 received a single 14 Gy fraction as a boost after external beam radiotherapy. The prostate was contoured on TRUS images acquired before (virtual US) and after (real US) needle implant by two radiation oncologists, whereas on MR prostate was independently contoured by the same radiation oncologists (MR1, MR2) and by a dedicated radiologist (MR3). Absolute differences of prostate volumes $(|\Delta \mathrm{V}|)$ and craniocaudal extents $(|\Delta \mathrm{dz}|)$ were evaluated. The Dice's coefficient (DC) was calculated to quantify spatial overlap between MR contours.

Results: Significant difference was found between $V_{\text {virtual }}$ and $V_{\text {live }}(p<0.001)$ for the first treatment fractions and between $V_{M R 1}$ and $V_{M R 2}(p=0.043)$. Significant difference between cranio-caudal extents was found between $\mathrm{d} \mathrm{z}_{\mathrm{virtual}}$ and $\mathrm{dz}_{\text {live }}(p<0.033)$ for the first treatment fractions, between $\mathrm{dz}_{\mathrm{virtual}}$ of the first treatment fractions and $\mathrm{dz}_{\mathrm{MR} 1}(p<0.001)$ and between $\mathrm{dz}_{\mathrm{MR} 1}$ and $\mathrm{dz}_{\mathrm{MR} 3}(p<0.01)$. Oedema might be responsible for some of the changes in US volumes. Average DC values resulting from the comparison MR1 vs. MR2, MR1 vs. MR3 and MR2 vs. MR3 were $0.95 \pm 0.04$ (range, $0.82-0.99$ ), $0.87 \pm 0.04$ (range, $0.73-0.91$ ) and $0.87 \pm 0.04$ (range, 0.72-0.91), respectively.

Conclusions: Our results demonstrate the importance of a multiprofessional approach to TRUS-guided HDR prostate brachytherapy. Specific training in MR and US prostate imaging is recommended for centers that are unfamiliar with HDR prostate brachytherapy.

J Contemp Brachytherapy 2018; 10, 4: 285-290 DOI: https://doi.org/10.5114/jcb.2018.77947
\end{abstract}

Key words: HDR prostate brachytherapy, MR imaging, TRUS-guided real-time treatment planning.

\section{Purpose}

High-dose-rate (HDR) brachytherapy (BT) as monotherapy or as a boost (i.e., combined with external beam radiotherapy) has been described in many prostate cancer trials showing a good clinical outcome and a favourable toxicity profile [1]. Ten-year long-term data of low- or intermediate-risk patients have proved a durable disease control in terms of biochemical disease-free survival and low toxicity [2]. According to mono-institutional studies with a median follow-up time up to 8 years, HDR-BT has also resulted in effective and safe in treating of intermediateor high-risk patients $[3,4]$.

Our HDR-BT experience started in December 2009 and was preceded by a preliminary period including attendance of both general and specific prostate courses as well as on-site visits to radiotherapy centres with long- 
time experience in BT (greater than 10 years). Concerning dose fractionation, we referred to Morton's and Martinez's experiences $[5,6]$. Our objective was to perform a limited number of implants (i.e., 1 implant for boosts, 2 implants for monotherapies), and remove needles after each treatment session. We chose trans-rectal ultrasound (TRUS) imaging with intra-operative on-line treatment planning [7], to have a real time vision of the needle implant and patient position without moving the patient to the computed tomography $(\mathrm{CT})$ or magnetic resonance (MR) scanner $[8,9]$ thus avoiding possible risk of implant dislocation.

TRUS imaging is used to define treatment feasibility (according to GEC ESTRO patient selection criteria, updated by Hoskin et al. [10,11]) and is essential for prostate, seminal vesicles, bladder, rectum, and urethra contouring for treatment planning. Nevertheless, at our institution, radiation oncologists (ROs) did not have any direct experience in ultrasound imaging and they underwent a preliminary coaching period of 3 months, once a week, with our ultrasound radiologists. Now, after a few years of experience, we decided to investigate the ability of our ROs to accurately contour the prostate on MR and TRUS images and to see how far they still are from the contouring performed by a dedicated radiologist.

The purpose of this study was to compare the contours of the prostate performed by two ROs and one radiologist on MR and TRUS images. TRUS intra- and inter-fraction variability as well as TRUS vs. MR inter-modality variability and inter-operator variability were studied.

\section{Material and methods}

\section{Patient and treatment characteristics}

Thirty patients affected by localized prostate cancer and treated with interstitial HDR prostate brachytherapy at the National Cancer Institute in Milan were included in this study. All the cases were proposed to brachytherapists after a multidisciplinary $1^{\text {st }}$ visit (including surgeon, radiation oncologist and medical oncologist) during which patients are presented with the various therapeutic options. Age of the participants ranged from 56 to 81 years; mean and median ages were 69 and 70 years, respectively. Distribution according to 2009 TNM stage ( $7^{\text {th }}$ edition) was as follows: T1c 23; T2a 3; T2b 3; T3a 1.

For staging purposes, all patients underwent endorectal coil MR imaging with the following sequence: fast spinecho T2-weighted $(1.5 \mathrm{~T})$, repetition time 3,360 ms, echo time $126 \mathrm{~ms}$, slice thickness $3 \mathrm{~mm}$, interslice gap $0.3 \mathrm{~mm}$.

All patients underwent transrectal ultrasound imaging at first visit in the BRT office for feasibility evaluation of the brachytherapy treatment. Patients eligibility criteria were selected according to the GEC ESTRO 2013 update [11]; in particular, patients were eligible if there were no problems with probe introduction (e.g. anorectal stenosis), if the visibility of prostate boundaries was adequate, if the pubic arch was not interfering with needles implantation, if prostate volume was below $60 \mathrm{cc}$ and if urethra anatomy was acceptable (e.g., no kinking or severe phimosis).

US imaging was performed with a Pro Focus Ultrasound System provided with TRUS probe (type 8848, BK Medical Systems, Herlev, Denmark). The TRUS probe was mounted on a stepper which allowed acquisition of axial images of the prostate every $1 \mathrm{~mm}$. A specific QA of the TRUS system is routinely performed at our hospital according to the AAPM TG-128 Report [12], and the CIRS 45 phantom (Computerized Imaging Reference Systems, Norfolk (VA), USA) is used to perform this task.

Twenty-five patients received an exclusive two-fraction (14 Gy / fraction) treatment, while the other 5 received a single 14 Gy fraction as a boost after external beam radiotherapy (50 Gy, 25 fractions). Eleven patients had neo-adjuvant androgen deprivation (NAAD) and 5 patients had adjuvant androgen deprivation (AAD). During each treatment fraction, patients underwent TRUS imaging both before (virtual US) and after needle implantation (live US) (Figure 1), according to the procedure of
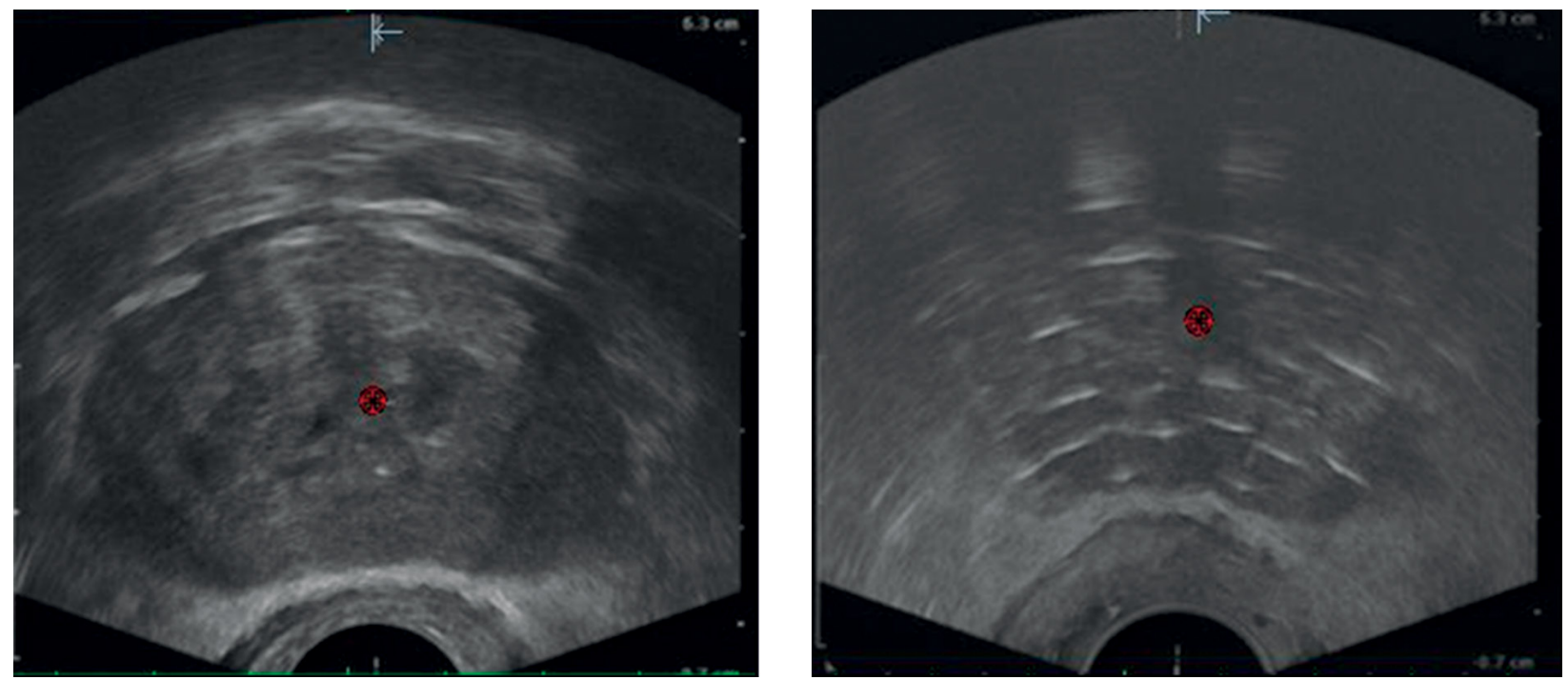

Fig. 1. Example of a virtual ultrasound image (i.e., before needle implantation) and live ultrasound image (i.e., after needle implantation) 
TRUS-based real-time treatment planning exhaustively described elsewhere [6]. The treatment was planned in real-time on the live US images and performed with a remote afterloader device provided with ${ }^{192} \mathrm{Ir}$ source (microSelectron HDR, Nucletron Elekta, Veenendaal, The Netherlands).

All cases were re-contoured for the study months to years after the actual treatment procedure by 2 ROs, separately. Live contours were made after virtual contours with an adaptation process, mimicking the actual treatment procedure.

\section{Evaluation and comparison of prostate volume and craniocaudal dimension}

Although the American Institute of Ultrasound in Medicine (AIUM) has developed practice parameter for the performance of an ultrasound evaluation of the prostate (and surrounding structures), to the best of our knowledge no specific guidelines are available for manual (i.e., non automated) prostate segmentation in HDR-BT [13]. Pelvic male normal tissue contouring guidelines for radiation therapy have been produced by the Radiation Therapy Oncology Group (RTOG) applying to CT scans [14]; prostate contouring errors and methods to improve prostate contouring accuracy were reported by Mc Laughlin et al. [15]. As for MR, an atlas of T2 MR prostate anatomy with CT correlation is available as a web resource [16]; some papers on prostate magnetic resonance imaging for brachytherapists were published recently by Soni, Venkatesan et al. $[17,18,19]$.

The prostate was contoured on US and MR images by means of the treatment planning system (TPS) Oncentra Prostate ver. 3.3 (Nucletron Elekta, Veenendaal, The Netherlands) and the MIM $^{\circledR}$ software (version 6.5.6, MIM Software Inc, Cleveland, Ohio, USA), respectively. As commonly done at our institution, US images were contoured by two radiation oncologists working together and providing one contouring result. On the contrary, MR images were independently contoured by the same two radiation oncologists, hereafter called MR1 and MR2. MR images were also contoured by a dedicated radiologist, hereafter MR3.

The volume $(V)$ and the cranio-caudal extent $(d)$ of the prostate were collected and compared. $V$ was automatically quantified on US and MR images by the TPS and the MIM software, respectively. On US images, $d$ was calculated multiplying the number of contoured images by the slice thickness. Whereas, on MR images, it was calculated multiplying the number of images by the slice thickness, including the gap between slices. The following contouring sets were compared (Figure 2): i) virtual vs. live US, for both fractions (i.e., TRUS intra-fraction comparison), ii) I fraction virtual/live vs. II fraction virtual/live US, in case of exclusive treatments (i.e., TRUS inter-fraction comparison); iii) MR1 vs. I fraction virtual US (i.e., intermodality comparison); iv) MR1 vs. MR2 (i.e., inter-operator comparison) and v) MR1/MR2 vs. MR3 (i.e., interprofessional comparison).

\section{Statistical analysis}

The statistical significance of the differences between volumes and cranio-caudal extents of different contouring sets was assessed with the Wilcoxon test for paired samples. A $p$-value $<0.05$ was considered significant. The test was performed using the software Statistica version 12 (StatSoft, Tulsa, OK, USA). Average and standard deviation of prostate volumes and of prostate cranio-caudal extents were also calculated for every set of contours.

MR1, MR2 and MR3 contours were superimposed in order to investigate in which prostate regions the differences between those contours were more considerable. In fact prostate volume alone is not a sufficient indicator of similarity: two contours could only partially overlap even if they have similar volumes. Dice's coefficients (DCs) were calculated for every combination of prostate contours drawn on MR images (MR1 and MR2, MR1 and MR3, MR2 and MR3) as a more specific indicator of similarity. Considering two volumes $A$ and $B, D C$ is defined as the intersection volume divided by the average volume, according to $\mathrm{DC}=2(\mathrm{~A} \cap \mathrm{B}) /(\mathrm{A}+\mathrm{B})$.

\section{Results}

Tables 1 and 2 show average prostate volumes $\langle V\rangle$ and cranio-caudal extents $\langle d\rangle$ calculated from the acquired images. The $p$-values for the different performed comparisons are shown. A significant difference was found between $\mathrm{V}_{\text {virtual }}$ and $\mathrm{V}_{\text {live }}(p<0.001)$ for the first treatment fractions, with $V_{\text {live }}>V_{\text {virtual }}$ in 23 out of 30 cases, and between $V_{\text {MR1 }}$ and $\mathrm{V}_{\mathrm{MR} 2}(p=0.043)$, with $\mathrm{V}_{\mathrm{MR} 1}>\mathrm{V}_{\mathrm{MR} 2}$ in 21 out of 30 cases. Regarding the cranio-caudal extents, a significant difference was found between $\mathrm{dz}_{\text {virtual }}$ and $\mathrm{dz}_{\text {live }}(p<0.033)$ for the first treatment fractions (i.e., $\mathrm{dz}_{\text {live }} \geq \mathrm{dz}_{\mathrm{virtual}}$ in 26 out of 30 cases), between $\mathrm{dz}_{\mathrm{virtual}}$ of the first treatment fractions and $\mathrm{dz}_{\mathrm{MR} 1}(p<0.001)$ with $\mathrm{dz}_{\mathrm{virtual}} \leq \mathrm{dz}_{\mathrm{MR} 1}$ in 27 out

\begin{tabular}{|c|c|c|c|c|c|}
\hline & Virtual TRUS & Live TRUS & MR1 & MR2 & MR3 \\
\hline $1^{\text {st }}$ fraction (overall: $25+5 \mathrm{pts}$ ) & $\longleftarrow$ & $\rightarrow$ & $\rightarrow \leftarrow$ & $\stackrel{4}{\longrightarrow}$ & $\begin{array}{l}\longrightarrow 5 \\
\longrightarrow 5\end{array}$ \\
\hline $1^{\text {st }}$ fraction (25 pts) & $2 \uparrow$ & $\uparrow_{2}$ & & & \\
\hline $2^{\text {nd }}$ fraction ( $\left.25 \mathrm{pts}\right)$ & $\downarrow \longleftarrow 1$ & $\downarrow$ & & & \\
\hline
\end{tabular}

Fig. 2. Schematic representation of the different comparisons between contouring sets performed in this study 
Table 1. Average prostate volumes $\langle\mathrm{V}\rangle$ with standard deviations $(k=1)$ and ranges calculated on ultrasound (US) and magnetic resonance images. The range of the differences between the analysed images is also provided. Regarding US images belonging to the first treatment fractions, average values were calculated both for all 30 patients and also for the subset of 25 patients who underwent brachytherapy as a two-fraction monotherapy treatment

\begin{tabular}{lccccc} 
Images & $n$ pts & $\langle\mathrm{V}\rangle\left(\mathrm{cm}^{3}\right)$ & Range $\left(\mathrm{cm}^{3}\right)$ & Statistical differences & Range of differences $\left(\mathrm{cm}^{3}\right)$ \\
\hline I fraction virtual US & 30 & $32.7 \pm 8.8$ & $13.4-56.3$ & & \\
\hline I fraction live US & 30 & $33.7 \pm 8.7$ & $13.5-56.6$ & $p<0.001(1 \mathrm{a})$ & $(-3.7-4.0)(1 \mathrm{a})$ \\
\hline I fraction virtual US & $25^{\star}$ & $34.4 \pm 8.0$ & $21.9-56.3$ & & \\
\hline I fraction live US & $25^{\star}$ & $35.5 \pm 7.8$ & $23.4-56.6$ & & \\
\hline II fraction virtual US & 25 & $34.0 \pm 7.0$ & $21.9-45.6$ & $p=0.58(2 \mathrm{a})$ & $(-10.7-10.3)(2 \mathrm{a})$ \\
\hline II fraction live US & 25 & $34.3 \pm 6.6$ & $23.0-48.0$ & $p=0.46(1 \mathrm{~b}) ; p=0.78(2 \mathrm{~b})$ & $(-3.1-6.0)(1 \mathrm{~b})$ \\
& & & & & $(-11.9-10.0)(2 \mathrm{~b})$ \\
\hline MR1 & 30 & $34.4 \pm 11.8$ & $14.4-77.0$ & $p=0.48(3)$ & $(-6.5-16)(3)$ \\
\hline MR2 & 30 & $33.9 \pm 10.9$ & $17.9-71.9$ & $p=0.043(4)$ &
\end{tabular}

*These 25 patients represent a subset of the 30 patients above, who underwent brachytherapy as a two-fraction monotherapy treatment and could be compared with the II fraction virtual and live US. (1a) - I fraction virtual vs. live US, (1b) - II fraction virtual vs. live US, (2a) - I fraction virtual vs. II fraction virtual US, (2b) - I fraction live vs. II fraction live US, (3) - MR1 vs. I fraction virtual US, (4) - MR1 vs. MR2, (5a) - MR1 vs. MR3, (5b) - MR2 vs. MR3. In bold, $p$ values smaller than 0.05. US - ultrasonography, MR - magnetic resonance

Table 2. Average prostate cranio-caudal extension $\langle d\rangle$ with standard deviations $(k=1)$ and ranges calculated on ultrasound (US) and magnetic resonance images. The range of the differences between the analysed images is also provided. Regarding US images belonging to the first treatment fractions, average values were calculated both for all 30 patients and also for the subset of 25 patients who underwent brachytherapy as a two-fraction monotherapy treatment

\begin{tabular}{lccccc} 
Images & $n$ pts & $\left\langle\mathrm{d}_{z}\right\rangle(\mathrm{mm})$ & Range $(\mathrm{mm})$ & Statistical differences & Range of differences $(\mathrm{mm})$ \\
\hline I fraction virtual US & 30 & $36.9 \pm 6.4$ & $26-55$ & & \\
\hline I fraction live US & 30 & $37.8 \pm 5.8$ & $27-55$ & $p=0.033(1 \mathrm{a})$ & $(-3-6)(1 \mathrm{a})$ \\
\hline I fraction virtual US & $25^{\star}$ & $37.6 \pm 6.5$ & $26-55$ & & \\
\hline I fraction live US & $25^{*}$ & $38.5 \pm 5.8$ & $30-55$ & & $(-6-10)(2 \mathrm{a})$ \\
\hline II fraction virtual US & 25 & $37.2 \pm 4.2$ & $29-48$ & $p=0.68(2 \mathrm{a})$ & $(-6-5)(1 \mathrm{~b})$ \\
\hline II fraction live US & 25 & $37.8 \pm 4.3$ & $29-48$ & $p=0.19(1 \mathrm{~b}) ; p=0.79(2 \mathrm{~b})$ & $(-8-8)(2 \mathrm{~b})$ \\
\hline MR 1 & 30 & $45.2 \pm 7.8$ & $29.7-62.7$ & $p<0.001(3)$ & $(-5.7-16.1)(3)$ \\
\hline MR 2 & 30 & $45.1 \pm 6.5$ & $33.0-59.4$ & $p=0.63(4)$ &
\end{tabular}

*These 25 patients represent a subset of the 30 patients above, who underwent brachytherapy as a two-fraction monotherapy treatment and could be compared with the II fraction virtual and live US. (1a) - I fraction virtual vs. live US, (1b) - II fraction virtual vs. live US, (2a) - I fraction virtual vs. II fraction virtual US, (2b) - I fraction live vs. II fraction live US, (3) - MR1 vs. I fraction virtual US, (4) - MR1 vs. MR2, (5a) - MR1 vs. MR3, (5b) - MR2 vs. MR3. In bold, $p$ values smaller than 0.05. US - ultrasonography, MR - magnetic resonance

of 30 cases, and between $\mathrm{dz}_{\mathrm{MR} 1}$ and $\mathrm{dz}_{\mathrm{MR} 3}(p<0.01)$, with $\mathrm{dz}_{\mathrm{MR} 1}>\mathrm{dz}_{\mathrm{MR} 3}$ in 16 out of 30 cases. It must be however noted that the analysis of difference between $\mathrm{dz}_{\mathrm{virtual}}$ and $\mathrm{dz}_{\mathrm{MR} 1}$ might be partially inaccurate, because the patient is unlikely to be in the same position for both MR and US scans, and the scanning plane is therefore unlikely to be the same.

Average DC values resulting from the comparison MR1 vs. MR2, MR1 vs. MR3 and MR2 vs. MR3 were $0.95 \pm 0.04$ (range 0.82-0.99), $0.87 \pm 0.04$ (range 0.73-0.91) and 0.87 \pm 0.04 (range 0.72-0.91), respectively. TRUS vs. MR intermodality comparison was not possible for one patient, who un- derwent hormonal therapy for prostate volume reduction after MR imaging and before the brachytherapy treatment.

\section{Discussion}

A trend towards higher live volumes in the first treatment fraction was observed. However, the number of cases in which volume differences are higher than $10 \%$ is limited. This means that the volumes contoured in the second part of the procedure (i.e., after needle implantation, therefore in a more difficult visual condition) are systematically bigger than those contoured in the first part of the procedure (i.e., on which the pre-planning is 
based), but the differences between the volumes are generally limited. The same trend was observed in the first treatment fraction for the cranio-caudal extent, with larger values for live contouring compared to those obtained in virtual contouring. Only in few cases, the difference was greater than $3 \mathrm{~mm}$.

As reported before, live contours were carried out starting from virtual image contours and adapting them to the new images. Even if a good initial overlap is possible by means of small rigid roto-translations, according to our experience it is necessary to review the target contour on every image in order to properly correct it to the new live image. It must be stressed that implanted needles produce image artifacts which make this task particularly difficult. In cases of doubt, a larger live volume is likely contoured by the observer as his/her conservative choice to not leave any region of the prostate uncovered by the treatment dose. Moreover, it is also well known that the prostatic gland might suffer moderate volumetric/dimensional modifications occurring during/after needle implantation. These modifications, which typically are its swelling caused by the edema produced by the needles, might have had an impact on the resulting difference between virtual and live targets [20,21].

With respect to the comparison between volumes and cranio-caudal extents drawn in the first and second treatment fractions, no significant differences resulted both in pre-planning and final contouring. Considering a mean time interval of about one month between first and second treatment fraction, this finding excludes an important prostate edema or at least its persistence in the second treatment fraction. This is in line with the results published for low-dose-rate brachytherapy treatments with permanent radioactive seeds, where prostate post-implant acute edema was found to resolve within about 1 month [22].

Prostate gland dissolves in the apex area and connects with the bladder wall in the base area. For this reason, even if US imaging is a very accurate imaging modality, it is undeniable that prostate contouring on US images is a demanding task. MR images available before intervention help to appreciate with greater accuracy the prostate anatomy, in particular those regions that are most difficult to be discriminated from the surrounding soft tissues on US images. Differences are predominant in the apex and the base regions $[23,24]$, but on the average they don't result in significant differences between the contoured volumes.

It must be however noted that US and MR images do not perfectly overlap because the prostate is differently deformed by the two endo-rectal probes. In fact, US and MR probes have different diameters (i.e., $2 \mathrm{~cm}$ for TRUS and up to $5 \mathrm{~cm}$ for MR), are used on patients positioned differently (i.e., lithotomy position for TRUS and prone position for MR) and provide different directional pressures. The prostate gland is therefore more compressed on MR images, with a reduced antero-posterior and an increased cranio-caudal extent. In addition to the different apex and base evaluation capability, this instrumental difference possibly explains the significantly longer cranio-caudal prostate extents on MR images. Nevertheless, differences between the two imaging modalities do not compromise the advantage of detailed anatomical information in MR images (e.g., apex and base structures, adenomatous prostatic hypertrophy, transurethral prostatic resections outcomes). The use of deformable image registration tools would probably help to improve quality of image registration $[24,25]$, however study of such tools was beyond the purposes of this work.

Comparison between contours drawn by two radiation oncologists on MR images aimed to investigate if prostate anatomy interpretation was similar between the two observers. It resulted that the first observer (MR1) identified a prostate volume that was significantly bigger than the one contoured by the second observer (MR2). Being however the average DC of the comparison between the two sets of volumes very high, it results that the overall prostate shapes identified by MR1 and MR2 are usually similar, with MR1 being less tight to the observed prostate edge than MR2. Interestingly, comparison with the contours drawn by a dedicated radiologist (MR3) resulted in differences between overall volumes that were not significant. However, local differences between the contours were appreciated, in particular in correspondence to the apex and base. Both radiation oncologists generally seemed to experience the same contouring difficulties and agreed more with each other (i.e., $\mathrm{DC}=0.95$ ) than with the radiologist (i.e., $\mathrm{DC}=0.87$ for both MR1 and MR2).

Figure 3 shows examples of possible critical regions of the prostate that were differently contoured by the three physicians. In general, radiologist identified shorter extensions of the prostate, with $\mathrm{d}_{\mathrm{MR} 3}$ being significantly lower than $\mathrm{d}_{\mathrm{MR} 1}$.
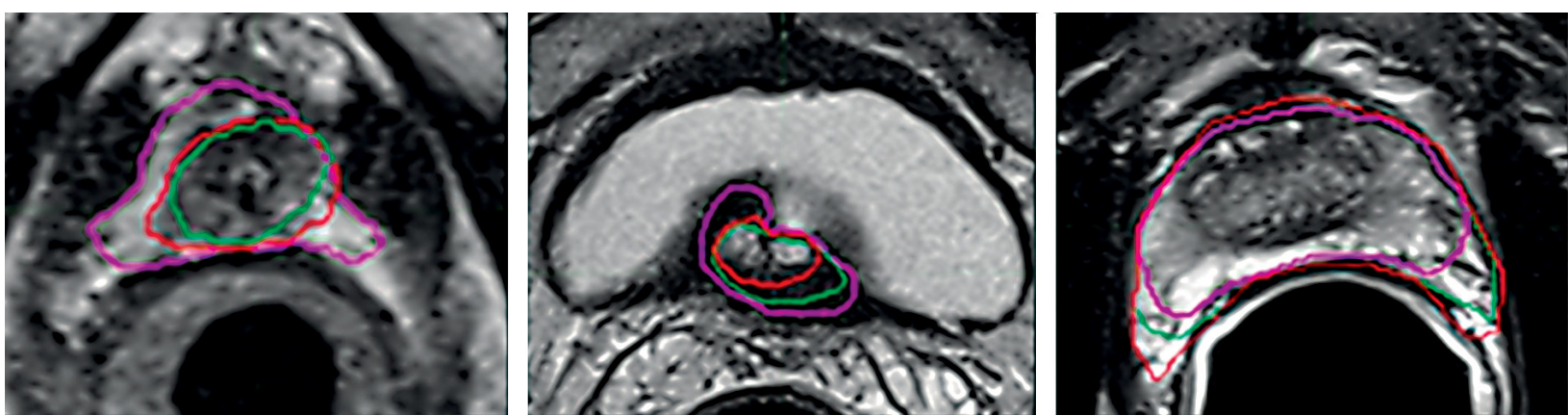

Fig. 3. Examples of regions of the prostate that were critical to be contoured accurately. From left to right: apex, base, and plexus. Red, green, and purple contours refer to MR1, MR2, and MR3, respectively 


\section{Conclusions}

No important volume differences resulted between the first and the second treatment fractions. The time gap between the two treatments was one month, apparently long enough for the resolution of possible post-implant acute edema. Also, virtual US and pre-implant MR volumes resulted relatively similar one to each other, despite different patient positioning and endorectal probes. The greater craniocaudal extents in MR images were most probably due to the stronger pressure on the prostate exerted by the endorectal coil.

Results of this study demonstrate the importance of a multiprofessional approach to TRUS-guided HDR-BT treatments of the prostate. A specific training in MR and US prostate imaging is recommended for centers that are not familiar with HDR prostate brachytherapy treatments. In the daily practice, even if radiation oncologists are expert in their field, we recommend to contour the pretreatment US and staging MR images and then to review them together with a radiologist, so to minimize contouring inaccuracies during the real treatment. Finally, in light of image quality detriment after the implant, due to needle artifacts, close attention is needed to adapt, slice by slice, virtual contours to live images.

\section{Disclosure}

The authors report no conflict of interest.

\section{References}

1. Thiruthaneeswaran N, Hoskin PJ. High dose rate brachytherapy for prostate cancer: Standard of care and future direction. Cancer Radiother 2016; 20: 66-72.

2. Hauswald H, Kamrava MR, Fallon JM et al. High-Dose-Rate Monotherapy for Localized Prostate Cancer: 10-Year Results. Int J Radiat Oncol Biol Phys 2016; 94: 667-674.

3. Yoshioka Y, Suzuki O, Isohashi F et al. High-Dose-Rate Brachytherapy as Monotherapy for Intermediate- and HighRisk Prostate Cancer: Clinical Results for a Median 8-Year Follow-Up. Int J Radiat Oncol Biol Phys 2016; 94: 675-682.

4. Blanchard P, Graff-Cailleaud P, Bossi A. Prostate brachytherapy: New techniques, new indications. Cancer Radiother 2018; 22: 352-358 [Article in French].

5. Morton GC, Loblaw DA, Sankreacha R et al. Single-fraction high-dose-rate brachytherapy and hypofractionated external beam radiotherapy for men with intermediate-risk prostate cancer: analysis of short- and medium-term toxicity and quality of life. Int J Radiat Oncol Biol Phys 2010; 77: 811-817.

6. Martinez AA, Demanes J, Vargas C et al. High-dose-rate prostate brachytherapy: an excellent accelerated-hypofractionated treatment for favorable prostate cancer. Am J Clin Oncol 2010; 33: 481-488.

7. Tselis N, Tunn UW, Chatzikonstantinou G et al. High dose rate brachytherapy as monotherapy for localised prostate cancer: a hypofractionated two-implant approach in 351 consecutive patients. Radiat Oncol 2013; 8: 115.

8. Tanderup K, Viswanathan AN, Kirisits C et al. Magnetic resonance image guided brachytherapy. Semin Radiat Oncol 2014; 24: 181-191.

9. D’Amico AV, Cormack R, Tempany CM et al. Real-time magnetic resonance image-guided interstitial brachytherapy in the treatment of select patients with clinically localized prostate cancer. Int J Radiat Oncol Biol Phys 1998; 42: 507-515.
10. Kovács G, Potter R, Loch T et al. GEC/ESTRO-EAU recommendations on temporary brachytherapy using stepping sources for localised prostate cancer. Radiother Oncol 2005; 74: 137-148.

11. Hoskin PJ, Colombo A, Henry A et al. GEC/ESTRO recommendations on high dose rate afterloading brachytherapy for localised prostate cancer: an update. Radiother Oncol 2013; 107: 325-332.

12. Pfeiffer D, Sutlief S, Feng W et al. AAPM Task Group 128: quality assurance tests for prostate brachytherapy ultrasound systems. Med Phys 2008; 35: 5471-5489.

13. The American Institute of Ultrasound in Medicine. AIUM Practice Parameter for the Performance of an Ultrasound Evaluation of the Prostate (and Surrounding Structures) - Parameter developed in collaboration with the American College of Radiology and the Society of Radiologists in Ultrasound. Available at: http:/ / www.aium.org/resources/ guidelines/prostate.pdf (Accessed: 30 October 2017).

14. Gay HA, Barthold HJ, O'Meara E et al. Pelvic normal tissue contouring guidelines for radiation therapy: A Radiation Therapy Oncology Group Consensus Panel Atlas. Int J Radiat Oncol Biol Phys 2012; 83: e353-e362.

15. McLaughlin PW, Evans C, Feng M et al. Radiographic and anatomic basis for prostate contouring errors and methods to improve prostate contouring accuracy. Int J Radiat Oncol Biol Phys 2010; 76: 369-378.

16. McLaughlin P, Troyer S, Berri S et al. Atlas of T2 MRI prostate anatomy with CT correlation. Available at: http:/ /www. prostadoodle.com/content/Prostate_Atlas_V3.pps.

17. Soni PD, Berlin A, Venkatesan AM et al. Magnetic resonance imaging-guided functional anatomy approach to prostate brachytherapy. Brachytherapy 2017; 16: 698-714.

18. Venkatesan AM, Stafford RJ, Duran C et al. Prostate magnetic resonance imaging for brachytherapists: Anatomy and technique. Brachytherapy 2017; 16: 679-687.

19. Venkatesan AM, Stafford RJ, Duran C et al. Prostate magnetic resonance imaging for brachytherapists: Diagnosis, imaging pitfalls, and post-therapy assessment. Brachytherapy 2017; 16: 688-697.

20. Carrara M, Tenconi C, Rossi G et al. In vivo rectal wall measurements during HDR prostate brachytherapy with MOSkin dosimeters integrated on a trans-rectal US probe: Comparison with planned and reconstructed doses. Radiother Oncol 2016; 118: 148-153.

21. Milickovic N, Mavroidis P, Tselis N et al. 4D analysis of influence of patient movement and anatomy alteration on the quality of 3D U/S-based prostate HDR brachytherapy treatment delivery. Med Phys 2011; 38: 4982-4993.

22. Liu D, Usmani N, Ghosh $S$ et al. Comparison of prostate volume, shape, and contouring variability determined from preimplant magnetic resonance and transrectal ultrasound images. Brachytherapy 2012; 11: 284-291.

23. Smith WL, Lewis C, Bauman G et al. Prostate volume contouring: a 3D analysis of segmentation using 3DTRUS, CT and MR. Int J Radiat Oncol Biol Phys 2007; 67: 1238-1247.

24. Daanen V, Gastaldo J, Giraud JY et al. MRI/TRUS data fusion for brachytherapy. Int J Med Robot 2006; 2: 256-261.

25. Reed G, Cunha JA, Noworolski S et al. Interactive, multi-modality image registrations for combined MRI/MRSI-planned HDR prostate brachytherapy. J Contemp Brachytherapy 2011; 3: $26-31$. 